\section{Venous anomalies as potentially lethal risk factors during ordinary catheterization}

\author{
Savino Occhionorelli, ${ }^{1}$ Sergio Gianesini, \\ Lorenzo Marinelli, ${ }^{2}$ Marianna Daniele, ${ }^{2}$ \\ Sara Chierici, ${ }^{2}$ Paolo Zamboni ${ }^{1}$ \\ ${ }^{1}$ Vascular Disease Center, University \\ of Ferrara; 'Department of Forensic \\ Medicine, University of Ferrara, Italy
}

\begin{abstract}
Venous malformations are rare but possible findings too, constituting a further risk factor for central venous catheter procedures. Herein we describe a case of death because of an innominate vein perforation by a catheter that incidentally was tucked into a sacciform malformation. Even if the technology advancement is constantly offering us new investigation tools, up to now diagnostic options are limited in the detection of those malformations that could potentially lead to dramatic complications as the described one. The present work raises the awareness about rare venous anomalies and their potential clinical implications. A proper literature review and diagnostic implementation proposal are reported.
\end{abstract}

\section{Introduction}

Nowadays, central venous access (CVA) is used on a daily basis worldwide. ${ }^{1}$ The Seldinger technique for the access has demonstrated to be safe and easy. ${ }^{2}$ Nevertheless, rare but possible mechanical severe complications have been reported. ${ }^{3}$ Venous malformations are rare but possible findings too, constituting a further risk factor for CVA-induced complications. ${ }^{4}$ We herein present an unlucky case of death because of a innominate vein perforation by a catheter that incidentally were tucked in a sacciform malformation. Related review of the literature is reported.

\section{Case Report}

A 77-year old female was scheduled for revision of an excessively shunting brachiocephalic dialysis-access. Her anamnesis reported atrial fibrillation with warfarin prophylaxis, dilatative and hypertensive right cardiomyopathy, Sjögren syndrome, chronic renal failure leading to a dialytic treatment since 7 years before.

A neck venous Doppler ultrasound (DUS) investigation was performed in order to evaluate the feasibility of CVA placement. The scanning revealed a right internal jugular vein (IJV) thrombosis and a physiological left venous district.

While waiting for the scheduled arteriovenous-fistula revision, after the administration of $500 \mathrm{IU}$ of human prothrombin complex because of a 2.01 international normalized ratio value, an echo-guided Quinton ${ }^{\mathrm{TM}}$ catheter (11 Fr; Covidien AG, Dublin, Ireland) placement was performed on the left IJV by means of a standard Seldinger technique. The echoguide was kept all along the vein poking and guidewire positioning, in order to verify the procedure correctness. The catheterization was performed by an expert anesthesiologist.

After the guide wire placement the catheter was introduced, finding a moderate resistance after just a few centimeters. The catheter was than retracted and easily positioned at the second try. The final correct catheter position was confirmed.

Some minutes later the patient begun to wag and to pale, suddenly turning pulseless but with a normal cardiac activity, as testified by the constant electrocardiogram monitoring. Despite immediate cardio-pulmonary resuscitation (CPR), asystole occurred. Atropine and adrenaline were administered, together with red blood cells.

Initially the patient responded, while an emergent X-ray demonstrated a massive hemothorax. The computed tomography revealed a left subclavian vein leakage on the posterior surface.

An emergent angiography was performed, detecting a bleeding from the left innominate vein. A VACS II Balloon Catheter (Osypka AG, Rheinfelden-Herten, Germany) was positioned in correspondence of the leaking point, so stopping the active bleeding.

Despite the procedure and the massive CPR (atropine 4 vials, adrenaline 6 vials, saline solution and colloids $6000 \mathrm{cc}$, red blood cells 8 IU to counteract a hemoglobin lowest level of $4.3 \mathrm{~g} / \mathrm{dL}$ ) the patient was declared dead $2 \mathrm{~h}$ after the CVA placement, without even the time to undergo an emothorax drainage.

The autopsy revealed an innominate vein venous malformation that was located $3.8 \mathrm{~cm}$ distally to the CVA insertion point, at $1.2 \mathrm{~cm}$ from the IJV-innominate vein confluence. The venous anomaly was a circular aneurismal like dilation, presenting a $0.9 \mathrm{~cm}$ diameter and a $0.7 \mathrm{~cm}$ depth. At its caudal margin a $0.3 \mathrm{~cm}$ long linear full-thickness perforation was detected (Figure 1).

The forensic scientist report analyzed the adverse event that occurred (the innominate vein rupture), pointing out both the unpredictability and inevitability of the fact. No fur-
Correspondence: Sergio Gianesini, Vascular Disease Center, University of Ferrara, via Aldo Moro 8, 44128 Cona (FE), Italy.

Tel.: +39.349.8012304.

E-mail: sergiogianesini@hotmail.com

Key words: Venous malformations; cerebral venous return; central venous catheters.

Received for publication: 8 March 2015.

Revision received: 24 April 2015.

Accepted for publication: 27 April 2015.

This work is licensed under a Creative Commons Attribution 3.0 License (by-nc 3.0).

(C)Copyright S. Occhionorelli et al., 2015

Licensee PAGEPress, Italy

Veins and Lymphatics 2015; 4:5098

doi:10.4081/vl.2015.5098

ther legal actions were undertaken.

\section{Discussion and Conclusions}

As testified by their worldwide use, CVA options have significantly improved the treatment chance and quality of a significant number of patients. ${ }^{3,4}$

Nevertheless, since it is very beginning, CVA use has been linked to rare but potentially severe complications. ${ }^{5}$

According to the literature, mechanical adverse events include arterial puncture (5\%), catheter malposition (4\%), catheter unsuccessful placement (4\%), subcutaneous hematoma (1\%), cardiac arrest (less than 1\%), pneumothorax (1\%), hemothorax (1\%). ${ }^{3,6}$

Aspirating blood from the catheter must be considered as not the only needed sign for correct placement: the catheter tip could be outside the vein, draining a blood collection caused by the vessel injury. For this reason infusion rates and diminished outflow from the catheter are adjunctive signs to be taken in immediate consideration.

Venous malformations represent an intriguing topic, often underestimated in its epidemiology. According to the literature this condition can be present as structural/morphological and hemodynamic/functional anomalies with an incidence of 1.2. ${ }^{7,8}$

As initially reported by Gadallah in 1995, awareness of venous malformations is a key element prior to whatever catheterization. ${ }^{4}$

As pointed out by this forensic scientist report, up to now the diagnostic options are unluckily limited in the detection of such increased risk elements, while no specific correlations have been reported among specific anamnesis and the described venous anomaly. 


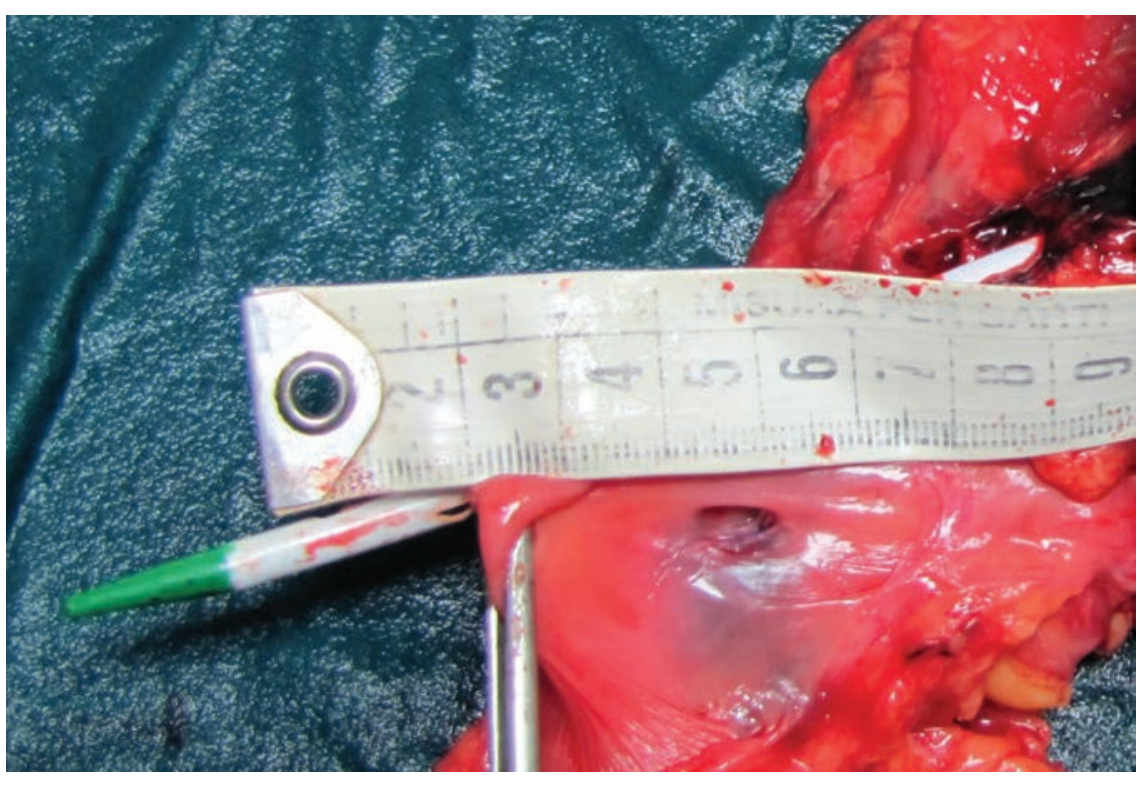

Figure 1. Innominate vein Doppler ultrasound-not detectable saccular malformation where the catheter tip were tucked in, causing the vessel perforation.

Nevertheless the technology advancement is constantly offering us new investigation tools. In this contest we can postulate that an emerging role of intra-venous ultrasound (IVUS) could happen in the near future.

This investigation technique is already available in the highly specialized vascular centers, where it demonstrated to be as effective as a traditional DUS in the detection of venous wall anomalies. ${ }^{9}$

The herein described case suggests the need of changing the inevitability and unpredictability of this kind of lethal adverse event in a so common procedure.

Surely not removing a not performing catheterization before further diagnostic assessments is the first advice to avoid immediate bleeding from an alternatively patent perforation. ${ }^{10}$

The awareness of the described possible vascular malformations opens a discussion regarding the emergent diagnostic protocols,
Committee; Centers for Disease Control and Prevention; 2011. Available from: http`/stacks.cdc.gov/view/cdc/5916/ Accessed: 6 February, 2014.

2. Higgs ZC, Macafee DA, Braithwaite BD, et al. The Seldinger technique: 50 years on. Lancet 2005;366:1407-9.

3. Eisen LA, Narasimhan M, Berger JS, et al. Mechanical complications of central venous catheters. J Intensive Care Med 2006;21:40-6.

4. Gadallah MF, White R, Vickers B, et al. Awareness of internal jugular, subclavian, superior vena cava and femoral venous anomalies may reduce morbidity of acute venous catheter procedures. Clin Nephrol 1995;44:345-8.

5. Apps MCP, Clark JMF, Skeater SJ. Hydrothorax, a complication of the insertion of central venous cannulae. Intens Care Med 1977;3:41-3.

6. Bardosi L, Mostafa SM, Wilkes RG, et al. Contralateral haemothorax: a late complication of subclavian vein cannulation. Br J Anaesth 1988;60:461-3.

7. Tasnadi G. Epidemiology and etiology of congenital vascular malformations. Semin Vasc Surg 1993;6:200-3.

8. Lee BB, Baumgartner I, Berlien P, et al. Diagnosis and treatment of venous malformations. Consensus document of the International Union of Phlebology (IUP): updated 2013. Int Angiol 2014 [Epub ahead of print].

9. Scalise F, Farina M, Manfredi M, et al. Assessment of jugular endovascular malformations in chronic cerebrospinal venous insufficiency: colour-Doppler scanning and catheter venography compared with intravascular ultrasound. Phlebology 2013;28:409-17.

1. O'Grady, Naomi P. Guidelines for the prevention of intravascular catheter-related infections, 2011. Atlanta, GA: Healthcare Infection Control Practices Advisory
10. Gibson F, Bodenham A. Misplaced central venous catheters: applied anatomy and practical management. Br J Anaesth 2013; 110:333-46. 